\title{
Bayesian Multiperiod Forecasting for Arma Model under Jeffrey's Prior
}

\author{
Zul Amry ${ }^{1, *}$, Adam Baharum ${ }^{2}$ \\ ${ }^{1}$ Department of Mathematics, State University of Medan, Indonesia \\ ${ }^{2}$ School of Mathematical Sciences, Universiti Sains Malaysia, Malaysia
}

Copyright $(\subset 2015$ by authors, all rights reserved. Authors agree that this article remains permanently open access under the terms of the Creative Commons Attribution License 4.0 International License.

\begin{abstract}
The main purpose of this study is to find the Bayesian forecast of ARMA model under Jeffrey's prior assumption with quadratic loss function. The point forecast model is obtained based on the mean of the marginal conditional posterior predictive in mathematical expression. Furthermore, the point forecast model of the Bayesian forecasting compared to the traditional forecasting. The simulation shows that the forecast accuracy of Bayesian forecasting is better than the traditional forecasting and the descriptive statistics of Bayesian forecasting are closer to the true value than the traditional forecasting.
\end{abstract}

Keywords ARMA Model, Bayes Theorem, Jeffrey's Prior, Multiperiod Forecast

\section{Introduction}

The Bayesian approach in general requires explicit formulation of a model and conditioning on known quantities in order to draw inferences about unknown ones. The main difference between the Bayesian approach and the classical approach is that in the Bayesian approach, the parameters supposed as random variables, which are described by their probability density function, whereas the classical approach considers the parameters to be fixed but unknown. The classical forecasting has been developed by Box and Jenkins [4]. There are three steps are accomplished in the process of fitting the ARMA $(p, q)$ model to a time series identification of the model, estimation of the parameters, and model checking to conclude whether the models obtained are adequate for forecasting. Several of works relating to Bayesian forecasting in the ARMA model are Fan \& Yao [8] and Uturbey [13] using ARMA with normal-gamma prior. Kleibergen \& Hoek [11], Liu [13], and Mohamed et al. [14] using ARMA model with Jeffrey's prior. This paper focuses on the Bayesian multiperiod forecasting for ARMA model using Jeffrey's prior with quadratic loss function.

\section{Materials and Methods}

The materials in this paper are a set time series data in the ARMA model for application and simulation. The method is study of literature by applying a set of theories of mathematical statistics such as the ARMA model, likelihood function, Jeffrey's prior, posterior distribution, Bayes theorem, conditional predictive density, conditional posterior predictive density, marginal conditional posterior predictive density, posterior mean and point forecast. Stages of discussion are presented in Figure 1 as follows:

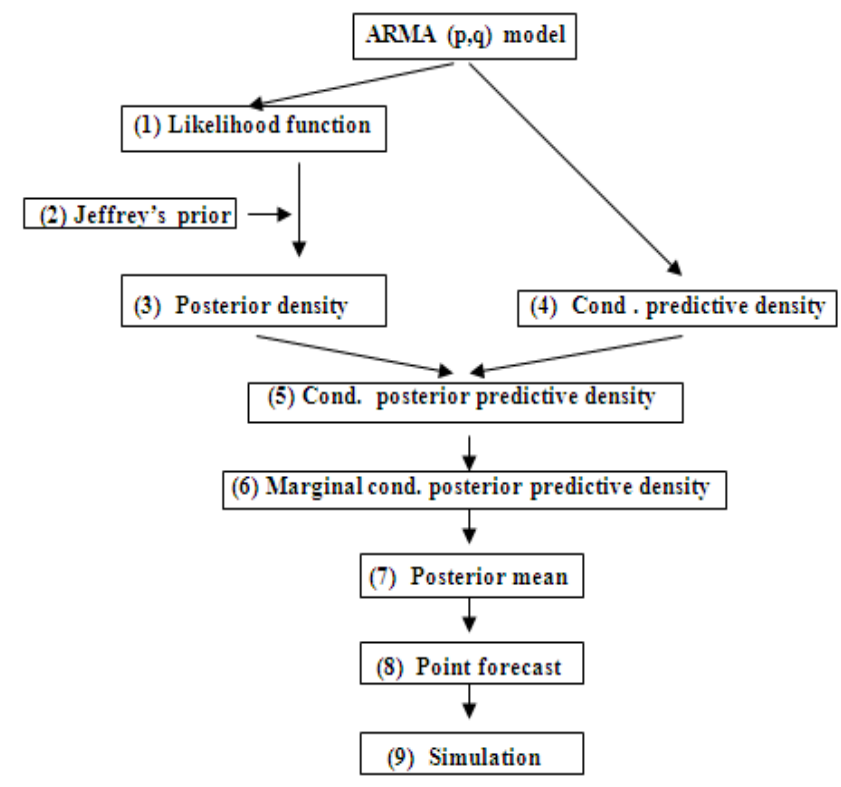

Figure 1. Stages of discussion

\section{Results}

\subsection{Likelihood Function}

The one-step-ahead point forecast of $y_{n+1}$ based data is observed $S_{n}=\left(y_{1}, y_{2}, \ldots, y_{n}\right)$ denoted by $\hat{y}_{n}(1)$, and defined 
by:

$$
\hat{y}(1)=E\left(y_{n+1} \mid S_{n}\right)
$$

This case is expandable for the $\mathrm{k}$-step-ahead point forecast of $y_{n+k}$, that is:

$$
\hat{y}(k)=E\left(y_{n+k} \mid S_{n}^{*}\right)
$$

where $S_{n}^{*}=\left(\mathrm{y}_{1}, \mathrm{y}_{2}, \ldots, \mathrm{y}_{\mathrm{n}+\mathrm{k}-1}\right)$

The ARMA $(p, q)$ model defined by:

$$
y_{t}=\sum_{i=1}^{p} \phi_{i} y_{t-i}+\sum_{j=1}^{q} \theta_{j} e_{t-j}+e_{t}
$$

where $\left\{e_{t}\right\}$ is sequence of $i$ id normal random variables with $e_{t} \sim N\left(0, \tau^{-1}\right), \tau>0$ and unknown, $\phi_{i}$ and $\theta_{j}$ are parameters.

Residuals are as:

$$
e_{t}=y_{t}-\sum_{i=1}^{p} \phi_{i} y_{t-i}-\sum_{j=1}^{q} \theta_{j} e_{t-j}
$$

By conditioning the first $\mathrm{p}$ observations and letting $e_{p}=e_{p-1}=\ldots=e_{r}=0$, where $\mathrm{r}=\min (0, \mathrm{p}+1-\mathrm{q})$, one may approximate ( Box \& Jenkins, [4] ), the likelihood function for $\Psi=\left(\phi_{1}, \phi_{2}, \ldots, \phi_{p}, \theta_{1}, \theta_{2}, \ldots, \theta_{q}\right)$ and $\tau$ based $S_{n}^{*}$ is :

$$
\exp \left\{-\frac{\tau}{2}\left[\sum_{t=p+1}^{n+k-1}\left(y_{t}-\sum_{i=1}^{p} \phi_{i} y_{t-i}-\sum_{j=1}^{q} \theta_{j} e_{t-j}\right)^{2}\right]\right\}
$$

The equation (3.5) can be expressed as:

$$
\times \exp \left\{-\frac{\tau}{2}\left[\sum_{t=p+1}^{L+k-1} y_{t}^{2}-2 \Psi^{T} \sum_{t=p+1}^{n+k-1} y_{t} \sum_{t=p+1}^{n+k-1}\left(\mathbf{B}^{*}+\Psi^{T} B_{t-1}\right)^{2}\right]\right\}
$$

where $B_{t}=\left(y_{t}, y_{t-1}, \ldots, y_{t+1-p}, e_{t}, e_{t-1}, \ldots, e_{t+1-q}\right)$

By allowing

$$
U=\left[\begin{array}{cccc}
y_{p} & y_{p+1} & \ldots & y_{n+k-2} \\
y_{p-1} & y_{p} & \ldots & y_{n+k-3} \\
\vdots & \vdots & \vdots & \vdots \\
y_{1} & y_{2} & \ldots & y_{n+k-1-p} \\
\hat{e}_{p} & \hat{e}_{p+1} & \ldots & \hat{e}_{n+k-2} \\
\hat{e}_{p-1} & \hat{e}_{p} & \ldots & \hat{e}_{n+k-3} \\
\vdots & \vdots & \vdots & \vdots \\
\hat{e}_{p+1-q} & \hat{e}_{p+2-q} & \ldots & \hat{e}_{n+k-1-q}
\end{array}\right]
$$

$$
x_{0}=\left[\begin{array}{c}
y_{p+1} \\
y_{p+2} \\
\vdots \\
y_{n+k-1}
\end{array}\right], W=U U^{T} \text { and } V=U x_{0}
$$

where $\hat{e}_{t}=y_{t}-\sum_{i=1}^{p} \widetilde{\phi}_{i} y_{t-i}-\sum_{j=1}^{q} \widetilde{\theta}_{j} \hat{e}_{t-j}, \mathrm{t}=\mathrm{p}+1, \mathrm{p}+2, \ldots, \mathrm{n}$, $\widetilde{\phi}_{i}$ and $\widetilde{\theta}_{j}$ are maximum likelihood estimator of $\phi_{i}$ and $\theta_{j}, \hat{e}_{t}, \hat{e}_{t-1}, \ldots, \hat{e}_{t-q}$ be obtained via:

$$
\hat{e}_{t}=y_{t}-\widetilde{\Psi}^{T} \boldsymbol{B}_{t-1}
$$

and the likelihood function can be expressed as:

$$
\propto \tau^{\left.((\mathrm{n}+\mathrm{k}-1)-\mathrm{p})\right|^{2}} \exp \left\{-\frac{\tau}{2}\left[\sum_{t=p+1}^{n+k-1} y_{t}^{2}-2 \Psi^{T} V+\Psi^{T} W \Psi\right]\right\}
$$

\subsection{Posterior Distribution under Jeffrey's Prior Assumption}

Based the likelihood function in equation (3.8), the Jeffrey's prior is:

$$
\pi_{\text {Jeff }}\left(\tau^{-1}\right) \propto \tau^{-1}
$$

By applying the Bayes theorem to equation (3.8) and (3.9), the posterior of $\Psi$ and $\tau^{-1}$ is:

$$
\begin{aligned}
& \pi\left(\Psi, \tau^{-1} \mid S_{n}^{*}\right) \propto \tau^{\frac{(n+k-1-2 p)+p}{2}-1} \times \\
& \times \exp \left\{-\frac{\tau}{2}\left[\Psi^{T} W \Psi-2 \Psi^{T} V+\sum_{t=p+1}^{n+k-1} y_{t}^{2}\right]\right\}
\end{aligned}
$$

\subsection{Conditional Predictive Density}

Based on $e_{t}=y_{t}-\sum_{i=1}^{p} \phi_{i} y_{t-i}-\sum_{j=1}^{q} \theta_{j} e_{t-j}$ with $e_{t} \sim N\left(0, \tau^{-1}\right)$, be obtained:

$$
f\left(e_{t} \mid S_{n}^{*}, \Psi, \tau^{-1}\right)=\left(2 \pi \tau^{-1}\right)^{-\frac{1}{2}} \exp \left\{-\frac{\tau}{2}\left[e_{t}\right]^{2}\right\} \text {, if }
$$

expressed in $y_{t}$ as:

$$
\begin{aligned}
& f\left(y_{t} \mid S_{n}^{*}, \Psi, \tau^{-1}\right)=\left(2 \pi \tau^{-1}\right)^{-\frac{1}{2}} \times \\
& \times \exp \left\{-\frac{\tau}{2}\left[y_{t}-\sum_{i=1}^{p} \varphi_{i} y_{t-i}-\sum_{j=1}^{q} \theta_{j} e_{t-j}\right]^{2}\right\}, \text { such }
\end{aligned}
$$

that the conditional predictive density of $Y_{n+k}$ based $S_{n}^{*}, \Psi$ and $\tau^{-1}$ is: 


$$
\begin{gathered}
f\left(y_{n+k} \mid S_{n}^{*}, \Psi, \tau^{-1}\right)=\left(2 \pi \tau^{-1}\right)^{-\frac{1}{2}} \times \\
\times \exp \left\{-\frac{\tau}{2}\left[y_{n+k}-\sum_{i=1}^{p} \varphi_{i} y_{n+k-i}-\sum_{j=1}^{q} \theta_{j} e_{n+k-j}\right]^{2}\right\} \\
\propto \tau^{\frac{1}{2}} \exp \left\{-\frac{\tau}{2}\left[y_{n+k}-\left(\sum_{i=1}^{p} \phi_{i} y_{n+k-i}+\sum_{j=1}^{q} \theta_{j} e_{n+k-j}\right)\right]^{2}\right\}(3.11)
\end{gathered}
$$

By changing $\sum_{i=1}^{p} \phi_{i} y_{n+k-i}+\sum_{j=1}^{q} \theta_{j} e_{n+k-j}$ to:

$$
\begin{aligned}
& \varphi_{1} y_{n+k-1}+\varphi_{2} y_{n+k-2}+\ldots+\varphi_{p} y_{n+k-p}+ \\
& +\theta_{1} e_{n+k-1}+\theta_{2} e_{n+k-2}+\ldots+\theta_{q} e_{n+k-q}
\end{aligned}=
$$$$
\left(\begin{array}{llllllll}
\phi_{1} & \phi_{2} & \ldots & \phi_{P} & \theta_{1} & \theta_{2} & \ldots & \theta_{q}
\end{array}\right)\left(\begin{array}{c}
y_{n+k-1} \\
y_{n+k-2} \\
\vdots \\
y_{n+k-p} \\
e_{n+k-1} \\
e_{n+k-2} \\
\vdots \\
e_{n+k-q}
\end{array}\right)=
$$

$$
\Psi^{T} B_{n+k-1}
$$

where $\quad B_{n+k-1}=\left(\begin{array}{l}y_{n+k-1}, y_{n+k-2}, \ldots, y_{n+k-p}, \\ e_{n+k-1}, e_{n+k-2}, \ldots, e_{n+k-q}\end{array}\right), \quad$ the equation (3.11) can be written as:

$$
\begin{gathered}
f\left(y_{n+k} \mid S_{n}^{*}, \Psi, \tau^{-1}\right) \propto \frac{1}{\frac{1}{2}} \\
\times \exp \left\{-\frac{\tau}{2}\left[y_{n+k}-\Psi^{T} B_{n+k-1}\right]^{2}\right\} \\
\propto \quad \frac{1}{2} \exp \left\{-\frac{\tau}{2}\left[y_{n+k}^{2}-2 \Psi^{T} B_{n+k-1} y_{n+k}+\Psi^{T} R \Psi\right]\right\}
\end{gathered}
$$

where $B_{n+k-1} \otimes B_{n+k-1}^{T}=R$ and $\left(\Psi^{T} B_{n+k-1}\right)^{2}=\Psi^{T} R \Psi$.

\subsection{Conditional Posterior Predictive Density}

Based equation (3.10) and equation (3.12) be obtained the conditional posterior predictive density:

$$
\begin{gathered}
f_{p}\left(y_{n+k} \mid S_{n}^{*}, \Psi, \tau^{-1}\right)^{\propto} \frac{(n+k-1-2 p)+p+1}{2}-1 \\
\times \exp \left\{-\frac{\tau}{2}\left[\begin{array}{c}
\Psi^{T} Z \Psi-\Psi^{T}\left(V+B_{n+k-1} y_{n+k}\right)- \\
\left(V^{T}+B_{n+k-1}^{T} y_{n+k}\right) \Psi+y_{n+k}^{2}+\sum_{t=p+1}^{n+k-1} y_{t}^{2}
\end{array}\right]\right\}
\end{gathered}
$$

where $\mathrm{Z}=\mathrm{W}+\mathrm{R}$

\subsection{Marginal Conditional Posterior Predictive Density}

The marginal conditional posterior predictive density of $Y_{n+k}$ be obtained by integrating equation (3.13) with respect to $\Psi$ and $\tau^{-1}$, that is:

$$
\begin{gathered}
f_{p}\left(y_{n+k} \mid S_{n}^{*}\right)=\int_{0}^{\infty} \int_{0}^{\infty} f_{p}\left(y_{n+k} \mid S_{n}^{*}, \Psi, \tau^{-1}\right) d \psi d \tau \\
\propto \int_{0}^{\infty} \tau \frac{y_{n+k}^{2}+\sum_{t=p+1}^{n+k-1} y_{t}^{2}-\left(V+B_{n+k-1} y_{n+k}\right)^{T} Z^{-1}\left(V+B_{n+k-1} y_{n+k}\right)}{2} \exp \left\{-\tau \times\left[\frac{[-2 p+p+1}{2}\right]\right\} d \tau
\end{gathered}
$$

By using the formula of gamma-distribution, based equation (3.14) be obtained:

$$
f_{p}\left(y_{n+k} \mid S_{n}^{*}\right) \propto\left[n+k-1-p+\left\{\frac{\left[y_{n+k}-\left(1-B_{n+k-1}^{T} Z^{-1} B_{n+k-1}\right)^{-1}\left(B_{n+k-1}^{T} Z^{-1} V\right)\right]^{2}}{\sum_{t=p+1}^{n+k-1} y_{t}^{2}-V^{T} Z_{0} V}\right\}\right]^{-\frac{(n+k-1-p)+1}{2}}
$$

The marginal conditional posterior predictive density of $Y_{n+k}$ is a univariate student's t-distribution on $(n+k-1-p)$ degrees of freedom with mean $\mu=\left(1-B_{n+k-1}^{T} Z^{-1} B_{n+k-1}\right)^{-1}\left(B_{n+k-1}^{T} Z^{-1} V\right)$. 


\subsection{Point Forecast}

For quadratic loss function, the point forecast of $Y_{n+k}$ is the posterior mean of the marginal conditional posterior predictive, that is:

$$
\begin{aligned}
& \hat{y}(k)=E\left(Y_{n+k} \mid S_{n}^{*}\right)= \\
& =\left(1-B_{n+k-1}^{T} Z^{-1} B_{n+k-1}\right)^{-1}\left(B_{n+k-1}^{T} Z^{-1} V\right)
\end{aligned}
$$

\section{Simulation}

A simulation study was conducted to compare between the Bayesian forecasting and the traditional forecasting of the ARMA $(1,1)$ model with $\widetilde{\phi}=0.93176$ and $\widetilde{\theta}=0.56909$ for series lengths of $70,90,110,130,150,180.210,240,270$ and 300 each to forecast the 10 steps ahead. The result of forecast by using the traditional method is:

$$
\begin{gathered}
\hat{y}(k)=\widetilde{\phi} \hat{y}_{60+k-1}+\widetilde{\theta} \hat{e}_{60+k-1}= \\
=0.93176 \hat{y}_{60+k-1}+0.56909 \hat{e}_{60+k-1}
\end{gathered}
$$

where $\hat{e}_{k}=\hat{y}_{k}-0.93176 \hat{y}_{k-1}-0.56909 \hat{e}_{k-1}$, $\mathrm{k}=1,2,3, \ldots, 10$.

\subsection{Comparison of Forecast Accuracy and Descriptive Statistics}

There are measures to determine the accuracy of a forecasting model. Assis [1] in their paper present four measures, namely Root Mean square Error (RMSE), Mean Absolute Error (MAE), Mean Absolute Percentage Error (MAPE) and U-statistic defined respectively as follows:

$$
\begin{aligned}
& R M S E=\sqrt{\frac{E S S}{n}} \\
& M A E=\frac{\sum_{t=1}^{n}\left|Y_{t}-\hat{Y}_{t}\right|}{n} \\
& M A P E=\frac{\sum_{t=1}^{n}\left|\frac{Y_{t}-\hat{Y}_{t}}{Y_{t}}\right|}{n} \times 100 \% \\
& U \text {-statistics }=\frac{R M S E}{\sqrt{\sum_{t=1}^{n} \frac{\hat{Y}^{2}}{n}}+\sqrt{\sum_{t=1}^{n} \frac{Y^{2}}{n}}}
\end{aligned}
$$

where $Y_{t}=$ the factual value at time $t, \hat{Y}_{t}=$ the forecast value at time $t, n=$ the number of observations, and $E S S=$ the error sum of square.

The comparison of forecast accuracy between Bayesian method in equation (3.16) with traditional method in equation (3.17) is presented in the Table 1. The comparison of descriptive statistics is presented in the Table 2, Columns 3 through 9 contain the minimum (Min), first quartile (Q1), median, mean, third quartile (Q3), maximum (Max), and standard deviation for $\mathrm{N}$ factual data, N-10 factual data and the result of Bayesian forecasting for the 10 steps ahead, and N-10 factual data and the result of traditional forecasting for the 10 steps ahead.

Table 1. Comparison of forecast accuracy

\begin{tabular}{|c|c|c|c|c|c|c|c|c|}
\hline \multirow{2}{*}{$\mathrm{N}$} & \multicolumn{9}{|c|}{ Bayesian } & \multicolumn{4}{c|}{ Traditional } \\
\cline { 2 - 9 } & RMSE & MAE & MAPE & U-STAT & RMSE & MAE & MAPE & U-STAT \\
\hline 70 & 1.2462 & 0.9816 & 0.1871 & 0.1239 & 1.6826 & 1.4202 & 0.3562 & 0.1501 \\
\hline 90 & 3.7513 & 3.2171 & 0.3578 & 0.2806 & 4.8687 & 4.1934 & 0.4677 & 0.3905 \\
\hline 110 & 0.6994 & 0.5659 & 0.7852 & 0.2188 & 0.8794 & 0.7826 & 0.6264 & 0.3084 \\
\hline 130 & 0.9393 & 0.7804 & 2.5616 & 0.3878 & 0.9455 & 0.7961 & 3.0440 & 0.3745 \\
\hline 150 & 1.1932 & 0.9763 & 0.1962 & 0.0847 & 1.5356 & 1.3354 & 0.2877 & 0.1068 \\
\hline 180 & 2.3678 & 1.9732 & 0.2687 & 0.1916 & 4.7827 & 3.5014 & 0.5130 & 0.3956 \\
\hline 210 & 2.0662 & 1.5278 & 0.2130 & 0.1884 & 3.2460 & 2.6855 & 0.3975 & 0.3314 \\
\hline 240 & 0.4899 & 0.3047 & 0.7064 & 0.3023 & 0.8176 & 0.7675 & 4.8140 & 0.3330 \\
\hline 270 & 0.4137 & 0.3818 & 1.5597 & 0.2640 & 0.6570 & 0.6069 & 1.0924 & 0.5617 \\
\hline 300 & 0.8170 & 0.7052 & 0.6543 & 0.4283 & 0.9923 & 0.9278 & 1.0468 & 0.3003 \\
\hline
\end{tabular}


Table 2. Comparison of descriptive statistics

\begin{tabular}{|c|c|c|c|c|c|c|c|c|}
\hline $\mathrm{N}$ & & Min. & Q1 & Median & Mean & Q3 & Max. & Stan. dev. \\
\hline \multirow{3}{*}{70} & Factual data & -7.147 & -4.289 & -1.322 & -0.9628 & 0.9795 & 8.493 & 4.009674 \\
\hline & Bayesian forecast & -7.147 & -4.289 & -1.322 & -0.9735 & 0.9795 & 8.493 & 4.011988 \\
\hline & Traditional forecast & -7.147 & -4.289 & -1.322 & -0.7801 & 0.9795 & 8.493 & 4.279630 \\
\hline \multirow{3}{*}{90} & Factual data & -4.65 & -2.057 & 0.7315 & 0.713 & 5.913 & 10.97 & 4.557475 \\
\hline & Bayesian forecast & -4.65 & -2.057 & 0.7315 & 0.355 & 4.955 & 8.424 & 4.097466 \\
\hline & Traditional forecast & -4.65 & -2.057 & 0.7315 & 0.247 & 4.470 & 8.424 & 4.019370 \\
\hline \multirow{3}{*}{110} & Factual data & -7.312 & -4.369 & -1.465 & -0.619 & 1.938 & 10.77 & 4.583494 \\
\hline & Bayesian forecast & -7.312 & -4.369 & -1.465 & -0.648 & 1.481 & 10.77 & 4.565613 \\
\hline & Traditional forecast & -7.312 & -4.369 & -1.465 & -0.680 & 1.242 & 310.77 & 4.552436 \\
\hline \multirow{3}{*}{130} & Factual data & -8.151 & -2.755 & -0.4623 & -0.2581 & 1.971 & 10.9 & 3.887779 \\
\hline & Bayesian forecast & -8.151 & -2.755 & -0.4623 & -0.2581 & 1.971 & 10.9 & 3.889712 \\
\hline & Traditional forecast & -8.151 & -2.755 & -0.4623 & -0.2680 & 1.971 & 10.9 & 3.881269 \\
\hline \multirow{3}{*}{150} & Factual data & -10.55 & -0.686 & 1.298 & 1.0190 & 3.362 & 10.46 & 3.963828 \\
\hline & Bayesian forecast & -10.20 & -0.686 & 1.298 & 0.9642 & 3.362 & 10.46 & 4.063910 \\
\hline & Traditional forecast & -10.17 & -0.686 & 1.298 & 0.9532 & 3.362 & 10.46 & 4.107567 \\
\hline \multirow{3}{*}{180} & Factual data & -8.273 & -2.465 & 0.3198 & 0.2020 & 2.994 & 8.262 & 3.915096 \\
\hline & Bayesian forecast & -7.341 & -2.465 & 0.3198 & 0.3078 & 2.994 & 8.262 & 3.742356 \\
\hline & Traditional forecast & -7.341 & -2.465 & 0.3198 & 0.3233 & 2.994 & 8.262 & 3.720305 \\
\hline \multirow{3}{*}{210} & Factual data & -11.97 & -6.403 & -3.571 & -2.955 & -0.535 & 8.739 & 4.760351 \\
\hline & Bayesian forecast & -11.97 & -6.403 & -3.571 & -3.025 & -0.535 & 8.088 & 4.626985 \\
\hline & Traditional forecast & -11.97 & -6.403 & -3.571 & -3.083 & -0.535 & 8.088 & 4.537499 \\
\hline \multirow{3}{*}{240} & Factual data & -4.269 & -1.011 & -0.0007 & 0.0034 & 0.9542 & 4.094 & 1.396144 \\
\hline & Bayesian forecast & -4.269 & -1.011 & 0.03994 & -0.006 & 0.8485 & 4.094 & 1.388480 \\
\hline & Traditional forecast & -4.269 & -1.011 & 0.03994 & 0.03275 & 1.055 & 4.094 & 1.414801 \\
\hline \multirow{3}{*}{270} & Factual data & -1.98 & 0.2856 & 1.191 & 1.186 & 1.89 & 5.231 & 1.309002 \\
\hline & Bayesian forecast & -1.98 & 0.2866 & 1.182 & 1.179 & 1.89 & 5.231 & 1.310926 \\
\hline & Traditional forecast & -1.98 & 0.2383 & 1.176 & 1.166 & 1.89 & 5.231 & 1.317332 \\
\hline \multirow{3}{*}{300} & Factual data & -3.527 & -1.104 & -0.02213 & -0.0013 & 0.9715 & 3.548 & 1.391662 \\
\hline & Bayesian forecast & -3.527 & -1.156 & -0.02213 & -0.0322 & 0.9715 & 3.548 & 1.380253 \\
\hline & Traditional forecast & -3.527 & -1.017 & -0.02213 & 0.02062 & 0.9715 & 3.548 & 1.426892 \\
\hline
\end{tabular}

\section{Conclusions}

The results in the Table 2 shows that the forecast accuracy value of the Bayesian method is smaller than the traditional method, it is indicates that the forecast accuracy the Bayesian forecasting is better than the traditional forecasting. The results in the Table 3 shows that descriptive statistics of Bayesian forecasting is closer to the factual data as compared to traditional forecasting, so it can be concluded that Bayesian forecasting is better than traditional forecasting.

\section{REFERENCES}

[1] Assis, K, Amran, A. and Remali, Y. (2010). Forecasting Cocoa Bean Prices Using Univariate Time Series Models.
Journal of Arts \& Commerce. ISSN 2229-4686, Vol.- I, Issue-I, 71-80

[2] Bain, L.J. and Engelhardt, M.(1992). Introduction to Probability and Mathematical Statistics, $2^{\text {nd }}$. Duxbury Press, Belmont, California.

[3] Bijak, J. (2010). Bayesian Forecasting and Issues of Uncertainty. Centre for Population Change, University of Southampton, 1-19.

[4] Box, G.E.P. and Jenkins, G. M. (1976). Time Series Analysis: Forecasting and Control. Holden-Day, San Francisco.

[5] Enders, W. (1995). Applied Econometric Time Series. John Wiley \& Son. Inc. New York.

[6] Faisal, F. (2012). Forecasting Bangladesh's Inflation Using Time Series ARIMA Models. Paper.

[7] Fan, C. and Yao, S. (2008). Bayesian Approach for ARMA 
Process and Its Application. International Business Research, 1(4), 49-55.

[8] Gelman, A. (2008), Objections to Bayesian statistics, Bayesian Analysis 3(3), 445-450.

[9] Geweke, J and Whiteman, C., (2004), Bayesian Forecasting, Department of Economics, University of Iowa.

[10] Ihaka, R. (2005). Time Series Analysis. Statistics Department University of Auckland.

[11] Kleibergen, F. and Hoek, H. (1996). Bayesian Analysis of ARMA model Using Non informative Prior. Paper, Econometric Institute, Erasmus University, Rotterdam, 1-24

[12] Liu,S. I.(1995). Bayesian Multiperiod Forecasts for ARX Models. Ann. Inst. Statist. Math. Vol. 47, no.2,211-224.

[13] Liu,S.I.(1995). Comparison of Forecast sfor AR Models Between A Random Coefficient Approach and A Bayesian Approach. Commun Statist.- Theory Meth., 24(2), 319-333

[14] Mohamed, I., Zaharim, A. and Yahya M.S. (2002). Penganggaran Parameter bagi Model BL (p,0,1,1) dengan Pendekatan Bayesian. Matematika, jilid 18, bil. 2, hlm. 129-136, Jabatan Matematika UTM.
[15] Pole, A., West, M. and Harrison, J. (1994). Applied Bayesian Forecasting and Time Series Analysis. Chapman and Hall, New York.

[16] Ramachandran, K.M. and Tsokos, C.P. (2009).Mathematical Statistics with Applications, Elsevier Academic Press. San Diego, California.

[17] Salam, M.A., Salam, S., and Feridun, M. (2006). Forecasting Inflation in Developing Nations: The Case of Pakistan. International Research Journal of Finance and Economics, ISSN 1450-2887, Issue 3, 138-159.

[18] Research Journal of Finance and Economics, ISSN 1450-2887, Issue 62, 111- 142

[19] Stovicek K. (2007). Forecasting with ARMA Models, The case of Slovenian Inflation, Prikazi in analize XIV/1, 23-45.

[20] Uturbey, W. (2006). Identification of ARMA Model by Bayesian Methods Applied to Streamflow Data. $9^{\text {th }}$ International Conference on Probabilistic Method Applied to Power Systems KTH (11-15June 2006, Stockholm, Sweden ), $1-7$

[21] Wei,W. W. S. (2006). Time Series Analysis Univariate and Multivariate Methods Addison Wesley Publishing Company, Inc. Canada. 